\title{
Part 4: Sport
}

\author{
Designing High-efficient and Easy-to-wear Ther- \\ mal Interface for Cooling of Wheelchair Athletes \\ During Training \\ Amaike Kakeru ${ }^{\mathrm{a}, \mathrm{b}}$, Jun Suzurikawa ${ }^{\mathrm{a}, *}$, Kennya \\ Kitagawa $^{\mathrm{a}, \mathrm{b}}$, Atsushi Takashima $^{\mathrm{a}}$, Yoshinori \\ Teshima $^{\mathrm{b}}$, Takenobu Inoue ${ }^{\mathrm{a}}$ and Toru Ogata ${ }^{\mathrm{a}}$ \\ ${ }^{a}$ National Rehabilitation Center for Persons with Dis- \\ abilities, 4-1, Namiki, Tokorozawa-shi, Saitama 359- \\ 8555, Japan \\ ${ }^{\mathrm{b}}$ Chiba Institute of Technology, 2-17-1, Tsudanuma, \\ Narashino-shi, Chiba 275-0016, Japan
}

Background: Heat accumulation is a well-known bottle neck of sport training for wheelchair athletes with cervical spinal cord injury (SCI), whose thermoregulatory system has serious dysfunction. However, there is no definitive method to continuously remove body heat during exercise on a wheelchair. The authors have proposed a neck cooling belt that is wearable in a sitting position on a racer wheelchair. The belt has a tubular structure made of flexible elastomer film, in which cold water is circulated. Although the positive effect of neck cooling during exercise was experimentally confirmed, the structure of the belt has not been optimized yet. In this study, two main components of the cooling belt, i.e. flow channel structure for water circulation and load-relief feature to reduce neck pressure due to tubing, were designed and evaluated in order to improve the usability.

Method: [Flow channel structure] For high-efficient heat transfer on the neck, the contact condition between the belt and skin is a key factor. The flexibility of the tubular belt changes depending on the film thickness, the pattern of heat seal and the inserts between the films for anti-obstruction. The influence and extent of these three design parameters were characterized by measurement of heat absorption rate (HAR) between the cooling belt and neck. [Load relief] Tubing from a coolant circulator hung on the wheelchair backrest to the cooling belt can cause unpleasant pressure on the neck during propulsion. To relieve this load, a shoulder strap was designed and prototyped. Considering usability for handling with paralyzed upper limbs, loopless and one-side structures were tested with measurement of pressure between the belt and neck skin.

Key results: [Flow channel structure] The comparison among elastomer films with the thickness of 100, 200, and $300 \mu \mathrm{m}$ showed that the decrease of HAR was measurable with $300-\mu \mathrm{m}$ one. The decrease of HAR was also observed to be induced by the heat-sealing pattern at the middle of the interface, which reduces the real contact area between the cooling belt and neck skin. Both patterned heat-sealing and the array of flow channel inserts were effective for anti-obstruction of cooling water when the belt was tightly worn for good contact to skin. With these results considered, the cooling belt made of the 200- $\mu \mathrm{m}$ film which has the insert array achieved HAR of $20 \mathrm{~W}$ with the cooling water of $18{ }^{\circ} \mathrm{C}$ in a hot environment of $31^{\circ} \mathrm{C}$. This HAR is almost twice as high as that reported previously. [Load relief] The measurement of the contact pressure between the cooling belt and neck skin showed the oneside shoulder strap caused unbalanced load on the neck due to its asymmetricity. The loop-less structure on the shoulder successfully relieved the load on the neck.

Conclusion: A neck cooling belt was designed and tested for thermoregulatory assist of wheelchair athletes. The parametric experiments quantified the relationship between HAR and flow channel structures and the effect of the loadrelief structure. The cooling belt with the optimal design achieved HAR of $20 \mathrm{~W}$ in average.

Keywords: Thermoregulation, Heat transfer, Neck cooling.

*Corresponding author. E-mail: suzurikawa-jun@ rehab.go.jp

Thermal characterization and field trial of a wearable coolant circulator for assist of thermoregulation in wheelchair athletes

Kennya Kitagawa ${ }^{\mathrm{a}, \mathrm{b}}$, Jun Suzurikawa ${ }^{\mathrm{a}, *}$, Kakeru 
Amaike ${ }^{a, b}$, Atsushi Takashima ${ }^{a}$, Toshiyo Tamura $^{c}$, Yukiharu Higuchi ${ }^{\mathrm{a}}$, Yoshinori Teshima ${ }^{\mathrm{b}}$, Takenobu Inoue $^{\mathrm{a}}$ and Toru Ogata ${ }^{\mathrm{a}}$

${ }^{a}$ National Rehabilitation Center for Persons with Disabilities, 4-1, Namiki, Tokorozawa-shi, Saitama 3598555, Japan

${ }^{\mathrm{b}}$ Chiba Institute of Technology, 2-17-1, Tsudanuma, Narashino-shi, Chiba 275-0016, Japan

${ }^{\mathrm{c}}$ Waseda University, 1-104, Totsukacho, Shinjuku-ku, Tokyo 169-8050, Japan

Background: Persons with spinal cord injury (SCI) have serious dysfunction in the thermoregulatory system. The loss of thermoregulatory responses in a hot environment, including sweating and vasodilation, sometimes leads to fatal incidents during intensive exercise. To overcome this problem, we have developed a thermoregulation assist system for wheelchair athletes. In a laboratory setting, neck cooling with a tubular belt in that cold water was circulated successfully suppressed the increase of core temperature in the subjects with cervical SCI during arm cranking. However, the effect of neck cooling in a real outer environment has not been demonstrated. In this study, we designed and prototyped a wearable coolant circulator that can be installed on a wheelchair. The thermal performance of the system was, then, characterized with benchmarking. Lastly, the system was used by wheelchair marathon athletes with cervical SCI under measurement of body temperatures.

Method: [System design] The coolant circulator consists of a Peltier device (max. heat absorption rate $=$ $40 \mathrm{~W}$ ), axial-flow pump, water reservoir, battery, and tubing to the neck belt. The size and weight of the system are $300(\mathrm{~W}), 200(\mathrm{H}), 100(\mathrm{D}) \mathrm{mm}$ and $2.9 \mathrm{~kg}$, respectively. To reduce the system volume, the water reservoir and the fitting components of tubing were specially designed and made by a $3 \mathrm{D}$ printer.

[Thermal characterization] The heat absorption from the neck belt and the environment into the coolant were measured by precise thermistors in an artificial climate chamber of $31^{\circ} \mathrm{C}$. The power consumptions of the Peltier device and the pump were also monitored in order to estimate the coefficient of performance (COP) of the system.

[Field trial] Two wheelchair marathon athletes were recruited as subjects of the field test. During running on the track on racer wheelchairs, the neck skin and core temperatures were monitored with and without cooling. The procedure of this trial was approved by the IRB of the National Rehabilitation Center for Persons with Disabilities, Japan.
Key results: [Thermal characterization] The maximum and average heat absorption rates at the neck were 48 and $15 \mathrm{~W}$, respectively. The heat loss out of the reservoir was $5 \mathrm{~W}$, while that out of the tubing was lower than the detection limit. Consequently, COP of the prototyped system was estimated at $40 \%$. This value is as high as that of the previously reported wearable cooling device.

[Field trial] During the 45-minute trial with cooling, the temperature of the skin above the carotid artery reached around $28.6^{\circ} \mathrm{C}$, and was $5.7^{\circ} \mathrm{C}$ lower in average than that in the non-cooling trial. The decrease of the core temperature by cooling was observed in one subject.

Conclusion: A wearable coolant circulator for neck cooling of wheelchair athletes was designed, prototyped and evaluated. The system can be installed on a racer-type wheelchair, and showed COP of $40 \%$ with the mean heat absorption rate of $15 \mathrm{~W}$. Cooling with the proposed system will be helpful for wheelchair users in outdoor activities as well as wheelchair athlete engaged in various types of sports.

Keywords: Neck cooling, Peltier device, Hot environment, Spinal cord injury, Wearable equipment.

* Corresponding author. E-mail: suzurikawa-jun@ rehab.go.jp

\section{Automatic Production System for Sports Program with Support Information}

Tsubasa Uchida ${ }^{\mathrm{a}, *}$, Hideki Sumiyoshi ${ }^{\mathrm{b}}$, Makiko Azuma $^{a}$, Naoto Kato ${ }^{\mathrm{a}}$, Shuichi Umeda ${ }^{\mathrm{a}}$, Nobuyuki Hiruma $^{\mathrm{b}}$ and Hiroyuki Kaneko ${ }^{\mathrm{a}}$

${ }^{a}$ Science \& Technology Research Laboratories, NHK (Japan Broadcasting Corporation), 1-10-11 Kinuta, Setagaya-ku, Tokyo, Japan

${ }^{\mathrm{b}}$ Advanced Technology Development and Research Division, NHK Engineering System, Inc., 1-10-11 Kinuta, Setagaya-ku, Tokyo, Japan

Background: For deaf and hard of hearing people viewing sports programs, commentary by Japanese Sign Language (JSL) helps them fully understand the situation of the game. To produce sports program with JSL is required the expertise in each sport to translate Japanese into JSL, and multiple interpreters need to stand by because the game mostly lasts for hours. However, the shortage of interpreters specialized in sports have become a barrier to increase the number of sports program with JSL. In order to solve these problem, we have developed an automatic production system for on- 
line distribution. When the situation difficult to understand without audio information such as penalty occurred, the system provides the detailed commentary by JSL computer graphics (CG) animation and subtitles in real time.

Method: The system automatically renders JSL CG animation from prepared templates of JSL phrases corresponding to fixed format match data distributed during sports game. Extracted items from match data such as players' names, scores, and penalties are inserted into templates of JSL sentences. After this, JSL CG animation is rendered automatically from completed sentences and JSL motion capture data that recorded phrase by phrase. The rendered JSL CG animation is outputted with Japanese subtitles and score information to the screen for each event. That screen output is overlapped with the game video sent over the Internet Protocol (IP) network and distributed as one streaming video. Finally, the live video stream is played on a web browser. The video player on a web browser has the push notification function that pops up text message on the game video area to alert the penalty event. If user clicks the pop-up text, the JSL video explaining that penalty in detail is automatically played. We also developed a video on demand (VOD) player diverting video generated by live streaming process. The VOD player has a seekbar with event icons referring to time-series event list automatically generated form match data.

Key results: We have confirmed that the system could render JSL CG animation and distribute overlapped video stream in real time. Compared with the previous method that needed time required to generate MP4 video files of JSL CG animation, about 30 seconds delay time from event occurrence to JSL CG animation display was decreased. Furthermore, the system could also adjust the synchronization between game video and JSL CG animation by giving buffer time to IP game video stream. Although the user needed to seek the specific scene by themselves when using previous VOD player, the new event timeline helped user to playback the scene for each event efficiently.

Conclusion: To improve the viewing experience of deaf and hard of hearing people, we have developed an automatic production system. The system enables to generate JSL CG animation, subtitles and score information automatically and distribute game video stream with that support information. As a result, sports programs with support information are produced without human resources. We will verify the effective support information and provision timing while applying our system to the various types of sports events.
Keywords: Japanese Sign Language, Computer Animation, Sports.

${ }^{*}$ Corresponding author. E-mail: uchida.t-fi@nhk.or.jp

First Evaluation of Information Support of everyone by everyone for everyone TimeLine (ISeeeTL) applied to Deaf and Hard of Hearing People Watching Sport

Rumi Hiraga ${ }^{\mathrm{a}, *}$, Daisuke Wakatsuki ${ }^{\mathrm{a}}$, Yuhki Shiraishi $^{\mathrm{a}}$, Masayuki Inoue ${ }^{\mathrm{a}}$, Yuka Kogo ${ }^{\mathrm{a}}$, Yoshiki Fukunaga $^{\mathrm{a}}$, Makoto Kobayashi ${ }^{\mathrm{a}}$, Manabi Miyagi ${ }^{\mathrm{a}}$, Takeaki Shionome $^{\mathrm{b}}$, Jianwei, Zhang ${ }^{\mathrm{c}}$ and Atsuyuki Morishima $^{\mathrm{d}}$

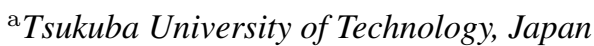

${ }^{\mathrm{b}}$ Teikyo University, Japan

${ }^{\mathrm{c}}$ Iwate University, Japan

${ }^{\mathrm{d}}$ University of Tsukuba, Japan

Background: Information support for deaf and hard of hearing $(\mathrm{DHH})$ people usually requires hearing people to provide support to $\mathrm{DHH}$ people. Information Support of everyone by everyone for everyone (ISeee) is a project that uses crowdsourcing to generate information support in which the support-giving role is not fixed; DHH people provide information based on their abilities and knowledge for hearing people and vice versa.

We are developing an ISeeeTimeLine (ISeeeTL) system which uses crowdsourcing to make watching sport more enjoyable. To find out what is required to enjoy watching sports (including parasports) with the ISeeeTL, we conducted experiments in which both hearing people and DHH people watched sports. The results focus on the DHH people because all the students we teach at a department of Tsukuba University of Technology are DHH.

Method: The ISeeeTL is a website (rather than an application) for posting about sports rules, results, teams, etc. It bears a slight resemblance to SNS applications like WhatsApp, but the ISeeeTL allows the posting of temporary comments besides posts. All the posts appear quicker than those on Twitter.

Fifty eight participants filled in questionnaires after each of the five games, 18 of whom were DHH people. There were 1852 posts, 349 of which were submitted by DHH participants. It took 456 minutes to watch the games. For some questions, they rated from one (strongly disagree) to 5 (strongly agree). We used the responses to find out whether there were any differences between the opinions of the two participant 
groups (DHH people and hearing people) and how ISeeeTL was appreciated when people were watching sports.

Key results: The questionnaires included three rating questions about reading the posts and four questions about writing the posts. We analyzed the answers to those questions with a two-way analysis of variance (ANOVA) in which the factors were the two participant groups and the questions.

The responses to the questions about reading posts showed there were significant differences between the groups $(p=0.02)$ and the questions $(p<0.01)$. DHH people evaluated reading posts better (mean 3.74) to hearing people (mean 3.36). The multiple comparison showed that the difference lies between the question on the usefulness of posts and the two questions on the quality and quantity of posts.

The responses to the questions about writing posts showed no difference between the two groups and a significant difference between the four questions $(p=0.01)$. The multiple comparison showed that the difference lies between the level of enjoyment from posting and those of the other three questions on burden, achievement, and importance of posting.

Conclusion: Though the current iteration of the ISeeeTL has received a positive evaluation on reading and writing posts, closer investigation shows that post quality and quantity should be improved. We are currently redesigning the ISeeeTL, and we are going to invite sports specialists to post valuable information.

The ISeeeTL has been officially approved as an information support system at some games of the National Athletics Meeting of Japan, including the wheelchair basketball games in 2019 .

Keywords: Deaf and Hard of Hearing, Information Support, Sports Watching, TimeLine, crowdsourcing. *Corresponding author. E-mail: rhiraga@a.tsukubatech.ac.jp 